\title{
Avaliação de Desempenho em Plantas Fotovoltaicas de Geração de Energia Elétrica
}

\author{
Dierli M. da R. Maschio ${ }^{1}$, Bruno Duarte ${ }^{1}$, Jean Patric da Costa $^{1}$, Marcelo Teixeira ${ }^{1}$ \\ ${ }^{1}$ Programa de Pós-Graduação em Engenharia Elétrica \\ Universidade Tecnológica Federal do Paraná \\ Pato Branco - PR - Brazil \\ \{dierli,brunoduarte\}@alunos.utfpr.edu.br, \{jpcosta,mtex\}@utfpr.edu.br
}

\begin{abstract}
In photovoltaic (PV) power systems, performance is a critical matter. In general, it is revealed after constructing and monitoring the real system, which fails to provide in-advance estimations that could benefit planning, dimensioning, and predictive maintenance. This paper proposes a Petri Net model that can quickly anticipate, with reasonable accuracy, the performance levels for PV systems with different sizes, architectures, generation profiles, and stages of construction. By receiving a set of offline specifications, the model can be exploited in advance, before actually constructing the real infrastructure to be measured. The proposed model is tested using a real PV plant and results show an accuracy of $93.4 \%$ in comparison with the real system.
\end{abstract}

Resumo. Em geração de energia fotovoltaica (FV), desempenho é um aspecto crítico, que pode ser revelado após a construção e o monitoramento do sistema real. Porém, isso inviabiliza a análise preditiva do sistema. Neste trabalho, propõe-se um modelo em Redes de Petri para antecipar, com razoável precisão, o desempenho de sistemas FV com diferentes tamanhos, arquiteturas, perfis de geração e estágios de implantação. Por receber um conjunto de especificações coletadas off-line, a abordagem permite explorar o sistema modelado sem efetivamente construí-lo ou medi-lo. A acurácia das estimativas providas é testada por meio de um experimento envolvendo uma planta $\mathrm{FV}$ real e os resultados mostram uma precisão de 93,4\% em comparação com o sistema real.

\section{Introdução}

Nos sistemas de geração de energia atuais, uma quantidade substancial de eletricidade ainda depende de fontes não renováveis, com alto teor de carbono [IEA 2018]. Nos últimos anos, no entanto, tem surgido um número considerável de fontes renováveis, que são formas eficazes e de baixa emissão de carbono para suprir, de forma sustentável, a rede de energia do futuro [Nehrir et al. 2011]. Dentre elas, a energia fotovoltaica (FV) tem se mostrado uma alternativa atrativa.

Uma questão importante em geração de energia $\mathrm{FV}$, é revelar possíveis flutuações que podem atrapalhar o desempenho do sistema [Jana and Chakraborty 2020, Olivares et al. 2014]. Essa habilidade permite aos gestores de energia negociar a produção a priori, seja para decidir sobre um possível armazenamento local, seja para negociar a venda do excedente para a rede elétrica principal, ou seja para complementar a energia gerada quando a produção local for insuficiente. 
As possíveis flutuações na geração podem ser facilmente reveladas monitorandose a infraestrutura real do sistema. No entanto, essa alternativa pressupõe que um sistema esteja em operação para ser medido, e, além disso, está longe de permitir a antecipação de informações para as etapas de planejamento, contingenciamento e correções preditivas. Essas etapas são mais beneficiadas por abordagens de modelagem e simulação que não dependem necessariamente da infraestrutura física [Paulista et al. 2019].

$\mathrm{Na}$ literatura, muitas abordagens têm se concentrado na modelagem de partes do comportamento contínuo de usinas de energia [Parvania and Scaglione 2015, Jana and Chakraborty 2020, Oyn Naversen et al. 2019]. Porém, alguns eventos (e.g., aqueles provenientes de sensoreamento, limitantes, violações) acontecem em um domínio discreto, e variações (e.g., perfil do usuário, de consumo, falhas) são essencialmente estocásticas, justificando complementos para o controle ativo e contínuo da rede elétrica [Cassettari et al. 2017, Ekyalimpa et al. 2016].

Em resultados anteriores [da R. Maschio et al. 2020], nós exploramos a hipótese de que um sistema de geração de energia pode ser visto, em certo nível de abstração, como um Sistema a Eventos Discretos (SED) [Cassandras and Lafortune 2008], para então ser modelado e controlado como tal, usando mecanismos baseados no design e no processamento de eventos.

Duas abordagens foram propostas e testadas, uma para a análise de disponibilidade, usando Redes de Petri [Simon et al. 2021], e outra mais focada no controle dos elementos ciber-físicos do sistema, nesse caso usando Máquinas de Estados Finitos [da R. Maschio et al. 2020]. Neste artigo, os resultados anteriores sobre análise de disponibilidade [Simon et al. 2021] de plantas FV são estendidos para incorporarem adicionalmente a avaliação de desempenho do sistema, o que é amparado pela proposta de um modelo baseado em Generalised Stochastic Petri Nets (GSPNs).

O modelo proposto pode ser diretamente combinado aos resultados anteriores e reconfigurado para projetar uma série de diferentes fluxos de trabalho em geração FV, além de incorporar o efeito encadeado das variáveis que normalmente oscilam em sistemas FV. Como o modelo é alimentado com parâmetros de especificações off-line, ele não depende do sistema FV real para ser simulado.

A fim de testar a abordagem proposta, foi avaliado o desempenho de uma planta FV real, instalada na UTFPR, em Pato Branco, PR, Brasil. Inicialmente, foi considerada a análise de um único painel $\mathrm{FV}$, o que é estendido em um segundo momento para um conjunto de 156 painéis. Para cada cenário, foram coletadas estimativas que visam antecipar como o sistema tende a responder a um conjunto de variabilidades impostas. As estimativas são então comparadas com os índices de desempenho do sistema real.

Os resultados sugerem que diversas métricas de interesse podem ser estimadas em um curto espaço de tempo, para sistemas FV com diferentes tamanhos, arquiteturas, perfis de geração e estágios de construção, o que acaba sendo uma ferramenta valiosa para engenharia preditiva de energia.

A seguir, a Seção 2 introduz os sistemas FVs e as Redes de Petri; a Seção 3 apresenta o modelo GSPN proposto; a Seção 4 descreve os experimentos e, por fim, a Seção 5 discute algumas conclusões e perspectivas futuras. 


\section{Fundamentação teórica}

Fontes de energia renovável desempenham um papel crucial para as redes de energia do futuro [IEA 2018], especialmente por permitirem reduzir a dependência de combustíveis fósseis e as emissões de gases no ambiente [Nehrir et al. 2011]. Dentre as diversas fontes renováveis, a energia solar fotovoltaica $(\mathrm{FV})$ tem se tornado popular devido à sua facilidade de manutenção, à natureza distribuída, e aos preços de instalação decrescentes.

Em uma usina FV, a eletricidade é produzida por células de paineis, sempre que estiverem expostas a radiação solar [Villalva et al. 2009, Yang et al. 2019]. Entretanto, sabe-se que a incidência solar é intermitente. Ao variar ao longo do dia, ela causa oscilação no desempenho da usina, tornando a qualidade da energia, o seu gerenciamento e a sua negociação, aspectos incertos [Denholm et al. 2010, King et al. 1997].

Uma possibilidade de antecipar a cadeia de impacto da intermitência solar na performance de uma usina FV, talvez assim contribuindo com a tomada de decisões de gestão, é modelando e simulando o comportamento da usina. Neste trabalho, utilizamos Redes de Petri para este propósito, as quais são introduzidas a seguir.

\subsection{Redes de Petri}

Uma Rede de Petri (RP) [Murata 1989] é um formalismo consolidado de modelagem que permite expressar e avaliar propriedades intrínsecas de Sistemas a eventos discretos (SEDs), como paralelismo, concorrência, exclusão mútua, etc. Enquanto o layout externo é intuitivo, similar a fluxogramas e diagramas de bloco, seu funcionamento interno implementa uma fundação matemática sólida, que permite a engenheiros expressar aspectos complexos de sistemas.

Estruturalmente, uma RP pode ser vista como um grafo direcionado composto por lugares, transições e arcos direcionados (conectando lugares e transições). Para expressar estados, bem como condições inerentes a estados, lugares são marcados com tokens. O estado de uma RP muda quando uma transição dispara, movendo tokens de lugares de entrada para lugares de saída, por meio dos arcos. Uma transição pode disparar se estiver habilitada, isto é, se seus lugares de entrada possuem tokens em número suficiente para que os arcos possam tomar. Além disso, o disparo pode depender de regras probabilísticas ou de prioridades associadas às transições.

Algumas extensões de RP têm sido desenvolvidas para incluir tempo e probabilidade em transições, permitindo a modelagem de outras classes de SEDs que evoluem dessa forma. Redes de Petri Estocásticas Generalizadas (acrônimo usual GSPNs) [Kartson et al. 1995], por exemplo, representam o tempo usando variáveis aleatórias associadas à transições temporizadas, que podem ser combinadas entre si ou com transições não temporizadas (ou imediatas) [Desrochers 1994]. Formalmente, uma GSPN é uma 7-tupla $\mathcal{N}=\langle\mathcal{P}, \mathcal{T}, \Pi, I, O, M, W\rangle$, em que:

- $\mathcal{P}=\left\{\rho_{1}, \rho_{2}, \cdots, \rho_{n}\right\}$ é um conjunto finito de lugares;

- $\mathcal{T}=\left\{\tau_{1}, \tau_{2}, \cdots, \tau_{n}\right\}$ é um conjunto finito de transições;

- $\Pi: \mathcal{T} \rightarrow \mathbb{N}$ é a função de prioridade, em que:

$$
\Pi(\tau)= \begin{cases}\geq 1, & \text { se } \tau \in \mathcal{T} \text { é uma transição imediata; } \\ 0, & \text { se } \tau \in \mathcal{T} \text { é uma transição temporizada; }\end{cases}
$$


- $I:(\mathcal{T} \times \mathcal{P}) \rightarrow \mathbb{N}$ é a relação de entrada que define os arcos que conectam lugares a transições;

- $O:(\mathcal{T} \times \mathcal{P}) \rightarrow \mathbb{N}$ é a relação de saída que define os arcos que conectam transições a lugares;

- $M: \mathcal{P} \rightarrow \mathbb{N}$ mapeia a marcação inicial, isto é, a quantidade de tokens em cada lugar $\rho \in \mathcal{P}$ (denotada \# $\rho$ );

- $W: \mathcal{T} \rightarrow \mathbb{R}^{+}$é a função de ponderação que representa tanto um peso $\left(w_{\tau} \in \mathbb{N}\right)$, quanto um atraso $\left(\lambda_{\tau} \in \mathbb{R}\right)$, tal que:

$$
W(\tau)= \begin{cases}w_{\tau} \geq 0, & \text { se } \tau \in \mathcal{T} \text { é imediata; } \\ \lambda_{\tau}>0, & \text { se } \tau \in \mathcal{T} \text { é temporizada. }\end{cases}
$$

A trajetória no espaço de estados de $\mathcal{N}$ depende das pré e pós condições de cada transição $\tau$, definidas respectivamente pelos conjuntos $\overleftarrow{\tau}=\{\rho \in \mathcal{P} \mid I(\tau, \rho)>0\} \mathrm{e}$ $\vec{\tau}=\{\rho \in \mathcal{P} \mid O(\tau, \rho)>0\} ; \tau$ é dita estar habilitada em uma marcação $M$ se, e somente se, $\forall \rho \in \overleftarrow{\tau}, M(\rho) \geq I(\tau, \rho)$. Portanto, um estado de $\mathcal{N}$ muda quando uma transição habilitada dispara e apenas transições habilitadas podem disparar. As imediatas disparam assim que são habilitadas, enquanto transições temporizadas aguardam um atraso $\lambda_{\tau}$, que pode ser tanto determinístico $\left(\lambda_{d \tau}\right)$, quanto estocástico $\left(\lambda_{s \tau}\right)$, sendo o primeiro caso associado a uma variável aleatória que modela alguma distribuição de probabilidade. Atrasos de transições temporizadas são inversamente proporcionais à frequência de disparo, relação esta que define a taxa de disparo da rede.

Uma importante característica de $\mathcal{N}$ diz respeito à sua limitação ou boundness. Quando uma transição dispara, ela move tokens dos lugares de entrada para os de saída. Portanto, o disparo de uma transição $\tau \in \mathcal{T}$, habilitada na marcação $M$, causa uma nova marcação $M^{\prime}$, tal que $\forall \rho \in(\overleftarrow{\tau} \cup \vec{\tau}), M^{\prime}(\rho)=M(\rho)-I(\tau, \rho)+O(\tau, \rho)$. $\mathcal{N}$ é dita ser limitada se permite um número finito $k>0$ de tokens em cada lugar, garantindo a finitude do espaço de estados resultante. Quando comparada com outras abordagens baseadas em espaço de estados, como as Cadeias de Markov, as GSPNs se mostram mais gerais, pois suportam tanto análises por simulação quanto no espaço de estados.

\section{Modelo de avaliação de desempenho proposto}

Em tese, um painel FV deve receber a irradiação solar, absorve-la e fornecer eletricidade. Entretanto, existem algumas variáveis neste processo que interferem no desempenho da geração de energia. Dentre elas: a irradiação solar é continuamente variável; cada painel possui uma capacidade constante e específica de produção; e a quantidade de energia a ser modelada e estimada deve ser associada a um determinado período de tempo, pois deseja-se interceptar essa estimativa em pontos discretos de tempo. Esses aspectos são absorvidos pela GSPN $P_{G}$, apresentada na Fig. 1.

Estruturalmente, o modelo $P_{G}$ é simples e intuitivo. A parte mais delicada está na forma como suas variáveis internas se relacionam. O modelo é composto por dois lugares, $\mathrm{R}$ e W, conectados pelas transições temporizadas $\tau_{1}$ e $\tau_{2}$. R quantifica os recursos (tokens) disponíveis para a geração de energia, enquanto $\mathrm{W}$ modela o estado gerando, ou seja, a energia que está sendo produzida. $\tau_{1}$ e $\tau_{2}$ impõem transições (mudanças) de estado em $P_{G}$ usando tokens em R e W, e disparando após os respectivos atrasos $\lambda_{1}$ e $\lambda_{2}$.

A transição $\tau_{1}$ dispara a uma taxa $1 / \lambda_{1}$, em que $\lambda_{1}$ é seu atraso, enquanto $\tau_{2}$ tem uma taxa de disparo de $1 / \lambda_{2}$. O atraso $\lambda_{2}$ também representa o tempo que o gerador leva 


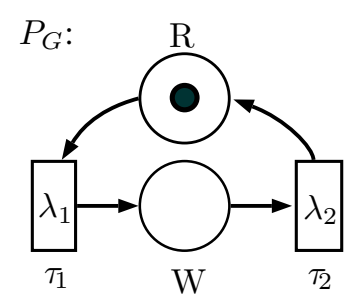

Figura 1. Modelo em GSPN para um gerador de energia FV.

para produzir uma determinada porção de energia. Neste artigo, definimos $M(\mathrm{R})=1$ em $P_{G}$, a fim de modelar geradores produzindo sequencialmente, uma unidade de energia por vez, após um atraso $\lambda_{2} \geq \lambda_{1}$.

Desta forma, diferentes potenciais de geração podem ser reproduzidos em um curto período de tempo simplesmente variando $\lambda_{2}$, ou configurando fluxos de trabalho que conectam vários modelos $P_{G}$. Isso permite capturar perfis distintos de plantas $\mathrm{FV}$, bem como mudanças climáticas e ambientais, como sombreamento dos painéis, oscilação de temperatura, intensidade do vento (quando for o caso), etc. Observe que esses eventos são reportados por sensores que se integram aos geradores no ambiente de produção e são usados para calcular parâmetros para $\lambda_{2}$, conforme a Eq. (1):

$$
\lambda_{2}=\frac{\Delta \mathrm{R}_{\mathrm{M}}}{\left(\mathrm{R}_{\mathrm{M}}-\min \left(\mathrm{R}_{\mathrm{M}}\right)\right)} \times T
$$

em que $\mathrm{R}_{\mathrm{M}}$ é o recurso real (medido); $\Delta \mathrm{R}_{\mathrm{M}}=\max \left(\mathrm{R}_{\mathrm{M}}\right)-\min \left(\mathrm{R}_{\mathrm{M}}\right)$ define a faixa viável de recursos que um gerador produz (neste caso, $\Delta R_{M}$ é a irradiação solar, então $\min \left(\mathrm{R}_{\mathrm{M}}\right)$ e $\max \left(\mathrm{R}_{\mathrm{M}}\right)$ representam, respectivamente, a irradiação mínima $\left(100 \mathrm{~W} / \mathrm{m}^{2}\right)$ e máxima (1000W/ $\mathrm{m}^{2}$ ) para a operação do painel); e $T$ é o intervalo de observação.

A seguir, esse modelo é simulado a fim de estimar algumas métricas que podem beneficiar e acelerar o processo de tomada de decisões de caráter preditivo na geração e gestão de energia. Por exemplo, pode ser interessante saber, para uma dada irradiação solar medida em alguma circunstância, qual é a infraestrutura necessária para entregar uma certa quantidade de energia de interesse? E, caso o sistema enfrente períodos de irradiação solar reduzida, qual seria a energia complementar a ser fornecida por outras fontes? Em caso de falha na usina, seja em um dado equipamento ou em uma string de painéis, o subsistema que permanece operando é suficiente para entregar o que é esperado?

Usando o modelo proposto, respostas para essas questões podem ser obtidas simplesmente variando-se os valores de $\lambda_{2}$ em $P_{G}$, conforme ilustrado na próxima seção.

\section{Resultados experimentais}

Esta seção mostra como o modelo proposto pode ser utilizado e o quão preciso ele se mostra para estimar métricas de desempenho em plantas FVs. Os experimentos a seguir são divididos em 4 subseções que descrevem respectivamente: a configuração física do sistema real a ser avaliado; como os parâmetros de entrada do modelo são definidos com base no sistema real (calibragem); o conjunto de simulações conduzidas; e a análise e discussão dos resultados. 


\subsection{Configuração do sistema físico}

Para alimentar o modelo com parâmetros de entrada e, posteriormente, comparar e validar suas estimativas, foi conduzido um estudo de caso envolvendo um sistema FV real, com $420 \mathrm{~kW}$ de potência máxima, instalado na Universidade Tecnológica Federal do Paraná (UTFPR), em Pato Branco, PR, Brasil. A Fig. 2 mostra o layout parcial da planta real.

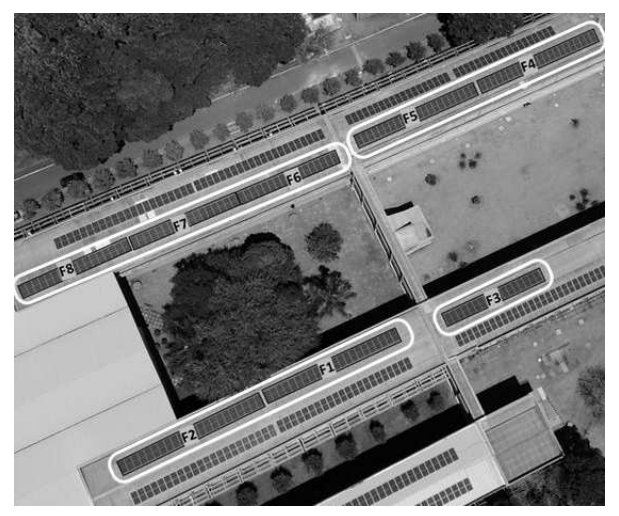

Figura 2. Layout parcial do sistema fotovoltaico da UTFPR.

A planta instalada, é composta por 1237 painéis, distribuídos em diferentes blocos de edifícios. Os painéis de cada bloco formam strings, que são centralizadas por inversores, responsáveis pela conversão de energia contínua em alternada. Cada string é conectada a um Maximum Power Point Tracker (MPTT), responsável por manter a máxima geração de energia proveniente dos painéis, através do controlador de corrente, mesmo em baixas tensões.

Para uma análise mais objetiva e clara do modelo proposto, o sistema real foi observado em duas etapas. Inicialmente, observou-se a operação de um único painel de $340 \mathrm{~W}$, o que acreditamos ser a análise de desempenho mais básica possível sobre um sistema FV. A observação das células do painel também é possível, entretanto ela incluiria um conjunto diferente de variáveis, o que não será considerado neste artigo. Em um segundo momento, após a validação do modelo para um único painel, a análise foi expandida para a observação de um inversor completo, composto por 8 strings que, juntas, instalam 156 painéis de $340 \mathrm{~W}$ cada, conforme detalhado na Tabela 1.

Tabela 1. Potencial de distribuição da planta.

\begin{tabular}{cccc} 
MPPT ID & String ID & Número de painéis & Potência Total $(W)$ \\
\hline MPPT 1 & $\mathrm{F}-1$ & 22 & 7480 \\
& $\mathrm{~F}-2$ & 22 & 7480 \\
MPPT 2 & $\mathrm{F}-3$ & 17 & 5780 \\
MPPT 3 & $\mathrm{F}-4$ & 22 & 7480 \\
& $\mathrm{~F}-5$ & 22 & 7480 \\
MPPT 4 & $\mathrm{F}-6$ & 17 & 5780 \\
& $\mathrm{~F}-7$ & 17 & 5780 \\
MPPT 5 & $\mathrm{F}-8$ & 17 & 5780 \\
\hline TOTAL & & 156 & 53040 \\
\hline
\end{tabular}




\subsection{Parâmetros de entrada}

A irradiação solar (denotada a seguir por $S\left(W / m^{2}\right)$ ) é reportada a cada 5 minutos por sensores instalados próximos a cada inversor do sistema. Esses dados são usados para calcular (pela Equação (1)) os parâmetros de entrada para o modelo GSPN da Fig. 1. O resultado é o conjunto de parâmetros detalhados na Tabela 2.

Tabela 2. Parâmetros de entrada para o modelo.

\begin{tabular}{|c|c|c|c|c|c|c|c|c|c|c|c|}
\hline Tempo & $S$ & $\tau_{2}$ & Tempo & $S$ & $\tau_{2}$ & Tempo & $S$ & $\tau_{2}$ & Tempo & $S$ & $\tau_{2}$ \\
\hline $07: 05$ & 106.3 & 514285.7 & $09: 45$ & 616.1 & 6277.8 & $12: 25$ & 807.1 & 4582.0 & $15: 05$ & 242.8 & 14490.1 \\
\hline $07: 10$ & 118.8 & 172340.4 & 09:50 & 629.9 & 6114.3 & $12: 30$ & 802.9 & 4609.4 & $15: 10$ & 607 & 5897.3 \\
\hline $07: 15$ & 124.2 & 133884.3 & $09: 55$ & 649.9 & 5891.9 & $12: 35$ & 806.8 & 4584.04 & $15: 15$ & 586.4 & 22689.0 \\
\hline $07: 20$ & 135.6 & 91011.2 & $10: 00$ & 660.5 & 5780.5 & $12: 40$ & 804.2 & 4600.9 & $15: 20$ & 541.3 & 6390.5 \\
\hline $07: 25$ & 166 & 49090.9 & $10: 05$ & 673.8 & 5646.5 & $12: 45$ & 800.2 & 4627.2 & $15: 25$ & 513.6 & 6661.1 \\
\hline $07: 30$ & 181.4 & 39803.4 & $10: 10$ & 684.1 & 5546.9 & $12: 50$ & 801.8 & 4616.7 & $15: 30$ & 507.6 & 7341.9 \\
\hline $07: 35$ & 150.2 & 64541.8 & $10: 15$ & 694.3 & 5451.7 & $12: 55$ & 797.1 & 4647.2 & $15: 35$ & 491.4 & 7833.6 \\
\hline $07: 40$ & 155.6 & 58273.3 & $10: 20$ & 703.9 & 5365.1 & $13: 00$ & 793.3 & 4673.3 & $15: 40$ & 489.9 & 7948.9 \\
\hline $07: 45$ & 185.5 & 37894.7 & $10: 25$ & 724.7 & 5186.4 & $13: 05$ & 791.4 & 4686.1 & $15: 45$ & 461.5 & 8277.9 \\
\hline $07: 50$ & 144 & 73636.3 & $10: 30$ & 726.5 & 5171.5 & $13: 10$ & 791.5 & 4685.4 & $15: 50$ & 447 & 8309.8 \\
\hline $07: 55$ & 164.9 & 49922.9 & $10: 35$ & 742.6 & 5042.0 & $13: 15$ & 793.7 & 4670.6 & $15: 55$ & 451.9 & 8962.6 \\
\hline 08:00 & 220 & 27000.0 & $10: 40$ & 750.6 & 4980.0 & $13: 20$ & 777.3 & 4783.7 & $16: 00$ & 240.7 & 9337.1 \\
\hline 08:05 & 180.5 & 40248.4 & $10: 45$ & 756.9 & 4932.2 & $13: 25$ & 753.1 & 4960.9 & $16: 05$ & 335.5 & 9207.1 \\
\hline $08: 10$ & 332.3 & 13947.4 & $10: 50$ & 764.1 & 4878.7 & $13: 30$ & 350.3 & 12944.4 & $16: 10$ & 498.6 & 23027.7 \\
\hline $08: 15$ & 347.8 & 13075.0 & $10: 55$ & 772.2 & 4819.9 & $13: 35$ & 760.9 & 4902.4 & $16: 15$ & 488 & 13757.9 \\
\hline $08: 20$ & 155.1 & 58802.1 & $11: 00$ & 778.3 & 4776.6 & $13: 40$ & 804.1 & 4601.6 & $16: 20$ & 453.9 & 8128.4 \\
\hline $08: 25$ & 368.4 & 12071.5 & 11:05 & 782.7 & 4745.8 & $13: 45$ & 776.6 & 4788.6 & $16: 25$ & 388.4 & 8350.5 \\
\hline $08: 30$ & 404 & 10657.8 & $11: 10$ & 788.7 & 4704.5 & $13: 50$ & 743.9 & 5031.8 & $16: 30$ & 425.8 & 9155.1 \\
\hline $08: 35$ & 397.9 & 10876.1 & $11: 15$ & 794 & 4668.5 & $13: 55$ & 588.1 & 6637.9 & $16: 35$ & 393.3 & 11234.4 \\
\hline $08: 40$ & 415.2 & 10279.1 & $11: 20$ & 799.7 & 4630.5 & $14: 00$ & 767.1 & 4856.8 & $16: 40$ & 342.3 & 9944.7 \\
\hline $08: 45$ & 427.5 & 9893.1 & $11: 25$ & 804.2 & 4600.9 & $14: 05$ & 770.4 & 4832.9 & $16: 45$ & 332 & 11046.7 \\
\hline $08: 50$ & 450.5 & 9243.9 & $11: 30$ & 806.4 & 4586.6 & $14: 10$ & 735.1 & 5101.5 & $16: 50$ & 291.2 & 13371.8 \\
\hline $08: 55$ & 457.6 & 9060.4 & $11: 35$ & 811.3 & 4555.0 & $14: 15$ & 757.9 & 4924.7 & $16: 55$ & 261.7 & 13965.5 \\
\hline 09:00 & 475.4 & 8630.7 & $11: 40$ & 817 & 4518.8 & $14: 20$ & 285.9 & 17428.7 & $17: 00$ & 258.2 & 16945.6 \\
\hline 09:05 & 503.3 & 8033.7 & $11: 45$ & 814.4 & 4535.2 & $14: 25$ & 644.5 & 5950.4 & $17: 05$ & 209.2 & 20037.1 \\
\hline 09:10 & 520.2 & 7710.6 & $11: 50$ & 819 & 4506.2 & $14: 30$ & 189.6 & 36160.7 & $17: 10$ & 186.4 & 20480.4 \\
\hline $09: 15$ & 530.9 & 7519.1 & $11: 55$ & 815.6 & 4527.6 & $14: 35$ & 161.4 & 52768.7 & $17: 15$ & 167 & 29670.3 \\
\hline $09: 20$ & 547.5 & 7240.2 & $12: 00$ & 817.7 & 4514.4 & $14: 40$ & 209.9 & 29481.3 & $17: 20$ & 151.9 & 37500.0 \\
\hline $09: 25$ & 569.9 & 6895.0 & $12: 05$ & 819.4 & 4503.7 & $14: 45$ & 635.6 & 6049.2 & $17: 25$ & 135.4 & 48358.2 \\
\hline $09: 30$ & 572.2 & 6861.4 & $12: 10$ & 814.6 & 4534.0 & $14: 50$ & 646.6 & 5927.5 & $17: 30$ & 121.8 & 62427.7 \\
\hline $09: 35$ & 595.9 & 6533.5 & $12: 15$ & 812.7 & 4546.0 & $14: 55$ & 323.6 & 14490.1 & $17: 35$ & 109.1 & 91525.4 \\
\hline $09: 40$ & 606.3 & 6399.3 & $12: 20$ & 821.7 & 4489.4 & $15: 00$ & 649.4 & 5897.3 & $17: 40$ & 98.9 & 148623.9 \\
\hline
\end{tabular}

O modelo calibrado é então simulado com o objetivo de antecipar a potência a ser eventualmente produzida pelos painéis. Todas as simulações neste artigo usam o algoritmo Stationary Simulation Standard implementado pela ferramenta TimeNet [Zimmermann and Knoke 2007], considerando um nível de confiança de $95 \%$, e erro relativo de $10 \%$.

Então, a taxa de disparo da transição $\tau_{2}$ foi variada, com o objetivo de estimar o Tempo de Resposta:

$$
\mathrm{RT}(\mathrm{W})=(\mathcal{E}(\mathrm{W})) \cdot\left(1 / \lambda_{2}\right),
$$

representando o tempo necessário para produzir certa quantidade de energia, em que

$$
\mathcal{E}(\mathrm{W})=(\# \mathrm{~W})
$$

captura a expectativa média de marcação no lugar W, enquanto RT confronta essa expectativa com a frequência de disparos, o retornando o tempo médio de espera em $\mathrm{W}$.

A variável Testá associada a 3600s, o que significa a geração de energia no período de uma hora. Quando $S \geq 1000 \mathrm{~W} / \mathrm{m}^{2}$, o atraso $\lambda_{2}$ é definido como $\lambda_{2}=3600$ 
$s$ que retorna a produção máxima de potência. Também $\lambda_{1}=3600 s$ é definido como o $\min \left(\lambda_{2}\right)$, visto que se pretende observar somente as variações em $\left(\lambda_{2}\right)$.

\subsection{Simulações}

As simulações a serem apresentadas a seguir também estão separadas em duas etapas, seguindo a forma como o sistema é observado na Seção 4.1. Na primeira etapa, foi simulada e estimada a potência produzida por um único painel $\mathrm{FV}$, enquanto a segunda etapa estima a quantidade de potência resultante do inversor contando os 156 painéis.

Observe que as estimativas para as strings de painéis têm uma natureza não aditiva em relação às estimativas de painel único, pois as condições que incidem sobre cada parte da string podem ser diferentes. Na verdade, é essa característica que retorna uma maior diversidade de análise sobre a quantidade real de potência resultante de um inversor.

Em nosso modelo, esse efeito não aditivo é capturado pela coleta de uma amostra de parâmetros de entrada para cada painel que compõe a string (semelhante à primeira etapa). Em seguida, os parâmetros de entrada são derivados para toda a string, com base na análise dos valores da média e do desvio padrão de cada amostra. Caso o desvio padrão seja significativo, a análise pode ser dividida por strings para isolar setores de produção mais instáveis. Caso contrário, o que é o caso dos experimentos a seguir, o desvio padrão pode ser desprezado, por ser ínfimo e não afetar a análise consideravelmente.

Para fins de validação, em ambas as etapas, as estimativas antecipadas pelo modelo são comparadas com a potência finalmente produzida pela planta real FV e reportada ao sistema de monitoramento por telemetria. Os resultados da comparação são mostrados na Fig. 3.

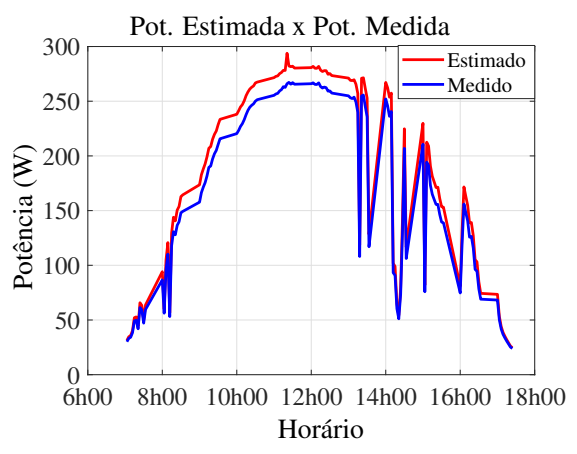

(a) Análise de 1 painel.

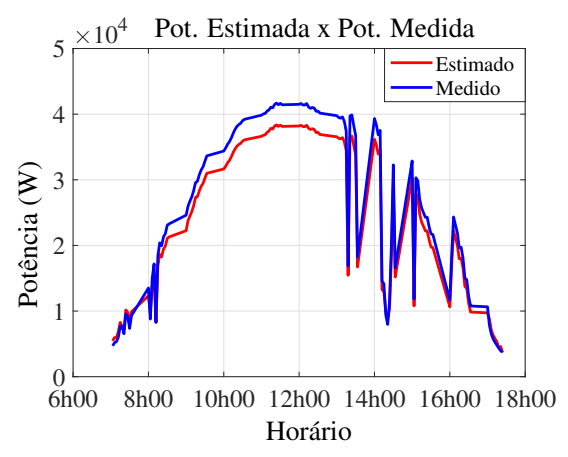

(b) Análise de 156 painéis.

Figura 3. Potência Estimada x Potência Medida de geração de energia.

\subsection{Análise dos resultados}

A comparação na Fig. 3 mostra que o modelo proposto estima o volume de geração de energia com precisão de aproximadamente $93,4 \%$, em ambos os testes, com relação ao desempenho real do sistema. Isso sugere que o modelo é preciso o suficiente para antecipar o fator de potência gerado por um painel ou uma série de painéis, absorvendo possíveis variabilidades internas.

Dessa forma, podemos responder à algumas questões postas anteriormente na Seção 3. Por exemplo, vamos supor que a usina tenha sido solicitada pelo sistema elétrico 
a atender a um consumo de $38 k W$ de energia, em média, entre às 10:00 e 13:00 horas. Espera-se saber se a geração dos 156 painéis, dentro desse intervalo e com as condições estimadas, é suficiente para atender a essa solicitação. A partir dos resultados da Fig. 3(b), podemos ver que a produção é estimada em aproximadamente $37 k W$, às 10:00 horas, e então $42.1 \mathrm{~kW}, 43.6 \mathrm{~kW}$ e $42 \mathrm{~kW}$, respectivamente às 11:00, 12:00 e 13:00 horas.

Nesse caso, o consumo é maior do que a produção às 10:00 horas, o que é revertido logo em seguida, às 11:00 horas. Isso significa que as equipes de engenharia e gestão podem ser informadas, a priori, que a energia possivelmente terá que ser complementada em alguns períodos, podendo gerar energia excedente e armazenamento em outros. Então, um banco de baterias poderia ser pensado para armazenar esse excedente e abastecer o sistema em períodos de baixa geração. Ou, a energia poderia ser negociada com o operador da rede elétrica principal, conforme conveniente. A relação entre banco de baterias, sobras, cargas e geração do sistema, é atualmente objeto de nossas pesquisas que estão em andamento.

Outra possibilidade de explorar os resultados e a usabilidade do modelo é expandir as análises para torná-las parte de um sistema maior de geração. Por exemplo, suponha que a usina FV avaliada seja parte de uma rede inteligente de escopo maior e integrada, que se concentra em fornecer energia cooperativamente via microrredes para a rede elétrica principal, por meio de um sistema de distribuição. Assuma, por exemplo, que esse sistema integrado possui a seguinte infraestrutura:

- $M^{1}$ é uma microrrede composta por 10 geradores eólicos de $6 \mathrm{~kW}$;

- $M^{2}$ é microrrede FV avaliada, composta de 156 painéis de $340 \mathrm{~W}$ cada;

- $M^{3}$ é uma Pequena Central Hidrelétrica $(\mathrm{PCH})$ de $500 \mathrm{~kW}$.

Agora, assuma que uma solicitação de geração foi recebida da rede elétrica principal, digamos às 11:30, para entregar um montante de $125.0 \mathrm{~kW}$ ao longo da próxima 1 hora. Nesse caso, qual deve ser a prescrição recomendada pelo operador do sistema para despachar essas três usinas?

Note que a própria PCH poderia prover a rede elétrica com a quantidade total de energia solicitada. No entanto, recursos hídricos devem ser economizados tanto quanto possível, dado a sua escassez, o impacto ambiental e a probabilidade de novas demandas de potência em um futuro próximo. Além disso, a água costuma ter um impacto encadeado ao longo do leito de um rio, sobre outras usinas e reservatórios de barragens, de maneira que as usinas hidrelétricas não podem ser despachadas arbitrariamente.

Portanto, a questão anterior pode ser reintroduzida da seguinte maneira: dada uma solicitação de $125.0 \mathrm{~kW}$ pela próxima 1 hora, qual seria a quantidade de energia produzida a partir de recursos eólicos e solares, que são renováveis, para que o operador do sistema possa despachar a PCH (ou outra fonte estável) apenas para preencher a demanda remanescente de geração?

Perceba que ainda não modelamos o desempenho da geração eólica, pois está fora do escopo deste artigo e talvez até seja um assunto para pesquisas futuras. Portanto, vamos supor que uma turbina eólica de $6 \mathrm{~kW}$ seja capaz de gerar cerca de $3,83 \mathrm{~kW}$ em uma hora, com uma incidência de vento de $5,4 \mathrm{~m} / \mathrm{s}$. Assim, de forma consistente com a realidade, assume-se que $M^{1}$ é capaz de entregar $38,3 \mathrm{~kW}$. 
Pelo nosso modelo, sabemos que $M^{2}$ entrega aproximadamente $42,0 \mathrm{~kW}$. Portanto, o montante remanescente a ser requisitado de $M^{3}$ é de $44,7 \mathrm{~kW}$.

Nesse cenário, as unidades eólica e fotovoltaica têm o potencial, respectivamente, de produzir aproximadamente $30,6 \%$ e $34,4 \%$ da energia solicitada. Portanto, a quantidade de energia que pode ser provida a partir de fontes renováveis é de 65, 0 \%. Assim, as fontes atuam de forma cooperativa para atender à demanda de geração solicitada.

\section{Conclusões}

Neste trabalho, os componentes de um sistema de energia FV são vistos como entidades dirigidas a eventos discretos que são modeladas utilizando GSPNs. A vantagem dessa abordagem reside no fato de que uma GSPN pode ser simulada para antecipar métricas de performance para sistema a eventos, o que a torna uma ferramenta poderosa para o planejamento de sistemas FV, desde a etapa de construção, de expansão de um sistema existente, ou para regular os contratos de comercialização no mercado de energia.

O modelo proposto foi testado sobre uma planta FV real. As estimativas mostram uma aderência de aproximadamente $90 \%$ em relação ao desempenho do sistema real. Pesquisas futuras e em andamento têm como meta estabelecer mecanismos para que as estimativas do modelo possam servir como apoio a sistemas de geração de energia cooperativos, no contexto de sistemas de energia ciber-físicos [SuperGrid 2021]. Também pretendemos testar nossa abordagem utilizando outros sistemas de geração de energia, como usinas eólicas.

\section{Agradecimentos}

Este trabalho teve suporte da Coordenação de Aperfeiçoamento de Pessoal de Nível Superior (CAPES), do Conselho Nacional de Desenvolvimento Científico e Tecnológico (CNPq), da Fundação Araucária (FA) e da Financiadora de Estudos e Projetos (FINEP).

\section{Referências}

Cassandras, C. G. and Lafortune, S. (2008). Introduction to Discrete Event Systems. Springer Science.

Cassettari, L., Bendato, I., Mosca, M., and Mosca, R. (2017). Energy Resources Intelligent Management using on line real-time simulation: A decision support tool for sustainable manufacturingl. Applied Energy, 190:841-851.

da R. Maschio, D. M., Mumbelli, J. D. C., Bonafin, A. C. T., and Teixeira, M. (2020). Supervisory Control of Distributed Power Generation Systems with Petri Net-based Customization. In International Workshop on Discrete Event Systems, pages 1-6, Rio de Janeiro, Brazil.

Denholm, P., Ela, E., Kirby, B., and Milligan, M. (2010). Role of Energy Storage with Renewable Electricity Generation. Technical report, National Renewable Energy Lab.(NREL), Golden, CO (United States).

Desrochers, A. A. (1994). Applications of Petri Nets in Manufacturing Systems: Modeling, Control and Performance Analysis. IEEE Press. 
Ekyalimpa, R., Werner, M., Hague, S., AbouRizk, S., and Porter, N. (2016). A combined discrete-continuous simulation model for analyzing train-pedestrian interactions. In IEEE Winter Simulation Conference (WSC), pages 1583-1594.

IEA, I. E. A. (2018). World energy balances: Overview. https://shorturl.at/fuL46.

Jana, D. and Chakraborty, N. (2020). Generalized stochastic Petri nets for uncertain renewable-based hybrid generation and load in a microgrid system. International Transactions on Electrical Energy Systems, 30(4):e12195.

Kartson, D., Balbo, G., Donatelli, S., Franceschinis, G., and Conte, G. (1995). Modelling with Generalized Stochastic Petri Nets. John Wiley \& Sons, Inc.

King, D. L., Kratochvil, J. A., and Boyson, W. E. (1997). Measuring solar spectral and angle-of-incidence effects on photovoltaic modules and solar irradiance sensors. In IEEE Conference on Photovoltaic Specialists, pages 1113-1116.

Murata, T. (1989). Petri Nets: Properties, Analysis and Applications. Proceedings of the IEEE, 77:541-580.

Nehrir, M., Wang, C., Strunz, K., Aki, H., Ramakumar, R., Bing, J., Miao, Z., and Salameh, Z. (2011). A review of hybrid renewable/alternative energy systems for electric power generation: Configurations, control, and applications. IEEE Transactions on Sustainable Energy, 2(4):392-403.

Olivares, D. E., Cañizares, C. A., and Kazerani, M. (2014). A centralized energy management system for isolated microgrids. IEEE Transactions on Smart Grid, 5(4):18641875.

Oyn Naversen, C., Farahmand, H., Helseth, A., and Catalão, J. (2019). Hydrothermal scheduling in the continuous-time framework. ArXiv, pages arXiv-1912.

Parvania, M. and Scaglione, A. (2015). Unit commitment with continuous-time generation and ramping trajectory models. IEEE Transactions on Power Systems, 31(4):3169-3178.

Paulista, C. R., Peixoto, T. A., and de Assis Rangel, J. J. (2019). Modeling and discrete event simulation in industrial systems considering consumption and electrical energy generation. Journal of Cleaner Production, 224:864-880.

Simon, D. F., Teixeira, M., and da Costa, J. P. (2021). Availability estimation in photovoltaic generation systems using Timed Petri Net simulation models. International Journal of Electrical Power and Energy Systems. Accepted for publication.

SuperGrid (2021). Supergrid institute: shaping power transmission. www.supergridinstitute.com.

Villalva, M. G., Gazoli, J. R., and Ruppert Filho, E. (2009). Comprehensive approach to modeling and simulation of photovoltaic arrays. IEEE Transactions on Power Electronics, 24(5):1198-1208.

Yang, H. E., French, R., and Bruckman, L., editors (2019). Durability and Reliability of Polymers and Other Materials in Photovoltaic Modules. William Andrew, 1 edition.

Zimmermann, A. and Knoke, M. (2007). TimeNET 4.0 - A Software Tool for the Performability Evaluation with Stochastic and Colored Petri Nets. 